\title{
Loss of the clock gene PER2 is associated with cancer development and altered expression of important tumor-related genes in oral cancer
}

\author{
HONGGANG XIONG, YIXIN YANG, KAI YANG, DAN ZHAO, HONG TANG and XIONGWEN RAN \\ Department of Oral and Maxillofacial Surgery, The First Affiliated Hospital \\ of Chongqing Medical University, Chongqing 400016, P.R. China
}

Received July 15, 2017; Accepted October 24, 2017

DOI: $10.3892 /$ ijo.2017.4180

\begin{abstract}
Recent studies have demonstrated that abnormal expression of the clock gene PER 2 is closely associated with the development of a variety of cancer types. However, the expression of PER2 in oral squamous cell carcinoma (OSCC), a common malignant tumor in humans, and its correlations with the clinicopathological parameters and survival time of OSCC patients and the altered expression of important tumorrelated genes remain unclear. In the present study, we detected the mRNA and protein expression levels of PER2, PIK3CA, PTEN,P53,P14ARF and caspase-8 in OSCC tissues and canceradjacent oral mucosa by reverse transcription-quantitative PCR (RT-qPCR), western blotting and immunohistochemistry. The results showed that the PER2, PTEN, P53, P14ARF and caspase- 8 mRNA and protein expression levels in OSCC were significantly reduced compared with those in cancer-adjacent tissues. Additionally, the PIK3CA protein expression level was significantly increased in OSCC tissues, whereas the mRNA level was not. Decreased expression of PER2 was significantly associated with advanced clinical stage and the presence of lymphatic metastasis in OSCC patients. Patients with PER2-negative expression had a significantly shorter survival time than those with PER2-positive expression. PER2 expression was negatively correlated with PIK3CA and P53 levels, and positively correlated with PTEN, P14ARF and caspase-8 levels. In summary, the results of this study suggest that loss of PER2 expression is closely associated with the genesis and development of OSCC and that PER2 may be an important prognostic biomarker in OSCC. PER2 may serve an antitumor role via the P53/P14ARF, PIK3CA/AKT and caspase-8 pathways.
\end{abstract}

Correspondence to: Professor Kai Yang, Department of Oral and Maxillofacial Surgery, The First Affiliated Hospital of Chongqing Medical University, 1 Youyi Road, Yuzhong, Chongqing 400016, P.R. China

E-mail: cqfyyk@hotmail.com

Key words: oral cancer, PER2, prognosis, tumor-related genes, correlation

\section{Introduction}

Clock genes exist in almost all cells of the human body $(1,2)$. They not only perform important roles in controlling the biological rhythm of the human body, but also affect cellular functions by regulating numerous genes. The downstream genes regulated by clock genes are called clock-controlled genes (CCGs) (3). CCGs are involved in various physiological activities, including cell proliferation, apoptosis, cell cycling, metabolism, endocrine signaling and immunity (4-8). As a result, abnormal expression of clock genes can lead to a variety of diseases, including endocrine disorders, cardiovascular diseases and tumors (9-12).

PER2 gene is an important clock gene. Changes in its expression are closely associated with the occurrence and development of cancers (13-17). Studies have demonstrated that PER2 expression levels are downregulated in multiple types of tumor, including gastric carcinoma, liver cancer, colon cancer, and head and neck squamous cell carcinomas (18-21). Furthermore, in vitro investigations of PER2 overexpression in lung cancer LLC cells, breast cancer EMT6 cells, osteosarcoma MG63 cells, and pancreatic cancer Pancl and Aspcl cells have demonstrated significant increases in tumor cell apoptosis and marked decreases in cell proliferation (22-24). Our previous studies have also shown that knockdown of PER2 in OSCC cells can greatly enhance cell proliferation and reduce apoptosis $(25,26)$. The aforementioned studies thus implicate PER2 as an important tumor-suppressor gene.

OSCC accounts for $90 \%$ of oral cancers, and is a common malignant tumor (27). At present, there is no clear opinion regarding the associations between PER 2 expression changes and the occurrence, development and survival outcomes of OSCC. It has been shown that the development of OSCC is closely related to expression changes of several important tumor-related genes, such as PIK3CA, PTEN, P53, P14ARF and caspase-8 (28-34). However, it is unclear whether there is any correlation between PER2 expression and the aberrant expression of these tumor-related genes.

In the present study, the mRNA and protein expression levels of PIK3CA, PTEN, P53, P14ARF and caspase-8 were assessed and compared between OSCC and cancer-adjacent 
Table I. Primary antibodies for detecting all target proteins.

\begin{tabular}{|c|c|c|c|c|c|}
\hline Primary antibodies & Brand & Catalog no. & Host & Applications & and dilutions \\
\hline PER2 & Novus & NBP2-24596 & Rabbit polyclonal & IHC-P:1:100 & \\
\hline PER2 & Abcam & $a b 208163$ & Rabbit polyclonal & & WB:1:250 \\
\hline PIK3CA & Bioworld & BS6052 & Rabbit polyclonal & IHC-P:1:200 & WB: $1: 1,000$ \\
\hline PTEN & CST & $9559 \mathrm{~T}$ & Rabbit monoclonal & IHC-P:1:160 & WB: $1: 1,000$ \\
\hline P53 & CST & $\# 2527$ & Rabbit monoclonal & IHC-P:1:160 & WB: $1: 1,000$ \\
\hline P14ARF & Novus & NB200-111 & Rabbit polyclonal & IHC-P:1:250 & WB: $1: 1,000$ \\
\hline Caspase- 8 & GeneTex & GTX110723 & Rabbit polyclonal & IHC-P:1:500 & WB: $1: 2,000$ \\
\hline GAPDH & CWBio & CW101M & Rabbit polyclonal & & WB: $1: 3,000$ \\
\hline
\end{tabular}

oral mucosa. In addition, the correlations between PER2 expression and clinicopathological parameters, survival time and expression levels of PIK3CA, PTEN, P53, P14ARF and caspase- 8 were analyzed, so as to provide a basis for further study into the function and mechanism of PER2 in cancer development.

\section{Materials and methods}

Antibodies. All primary antibodies used in the study were rabbit anti-human antibodies, and details are provided in Table I. Horseradish peroxidase (HRP)-conjugated goat anti-rabbit IgG secondary antibodies were purchased from CWBio Biotechnology (Beijing, China).

Patients and specimens. Fresh OSCC tissues and canceradjacent tissues were obtained from 8 patients with OSCC who underwent surgery at the First Affiliated Hospital of Chongqing Medical University (Chongqing, China) between September 2016 and November 2016. The clinicopathological data of these 8 OSCC patients are shown in Table II. In addition, paraffin-embedded tissue sections of 40 OSCC patients were obtained from the Pathology Department of the First Affiliated Hospital of Chongqing Medical University for immunohistochemical staining. The 40 patients were in-patients treated at the Department of Oral and Maxillofacial Surgery in the First Affiliated Hospital of Chongqing Medical University between 2007 and 2011; the clinicopathological data of these 40 patients are presented in Table III. From the 40 paraffin-embedded sections, 14 cancer-adjacent oral mucosa specimens were selected and used as the control group. None of the patients underwent radiotherapy or chemotherapy prior to surgery. The study was approved by the Biomedical Ethics Committee of the First Affiliated Hospital of Chongqing Medical University (approval no. 2016-124) and written informed consent was obtained from all patients who participated.

Reverse transcription-quantitative PCR (RT-qPCR). A liquid nitrogen grinding method was used to prepare the fresh OSCC and cancer-adjacent oral mucosa tissues from 8 patients. Total RNA was extracted using TRIzol reagent (Takara, Shiga, Japan) according to the manufacturer's instructions. A PrimeScript ${ }^{\mathrm{TM}}$ reagent kit with gDNA Eraser (Takara) was used to synthesize cDNA by reverse transcription. The primers for the PER2, PIK3CA, PTEN, P53, P14ARF and caspase-8 genes, as well
Table II. Clinicopathological features of 8 patients with OSCC.

Parameters

Cases

Tissue type

ANT 8

OSCC 8

Sex

Male 6

Female 2

Age

$\geq 60$

$<60$

Tumor differentiation

Poor 5

Moderate and well

TNM staging

$\mathrm{T} 1+\mathrm{T} 2$ 2

$\mathrm{T} 3+\mathrm{T} 4$

Lymph node metastases

Yes

No

Clinical stage

I+II 2

III+IV

Site

Gingiva

1

Tongue

3

Buccal 3

The floor of the oral

ANT, adjacent non-cancerous tissues. OSCC, oral squamous cell carcinoma.

as GAPDH, were designed with Oligo 7.0. The forward and reverse primer sequences for each gene are shown in Table IV. The reaction system included $12.5 \mu 12 \mathrm{X}$ SYBR Premix Ex $\mathrm{Taq}^{\mathrm{TM}}$ II, $1 \mu \mathrm{l} 0.4 \mu \mathrm{M}$ forward primer and reverse primer, $2 \mu \mathrm{l}$

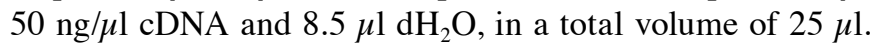
A C- $1000^{\mathrm{TM}}$ Thermal Cycler (Bio-Rad, CA, USA) was used 
Table III. Clinicopathological features of 40 patients with OSCC.

\begin{tabular}{|c|c|}
\hline Parameters & Cases \\
\hline \multicolumn{2}{|l|}{ Tissue type } \\
\hline ANT & 14 \\
\hline OSCC & 40 \\
\hline \multicolumn{2}{|l|}{ Sex } \\
\hline Male & 25 \\
\hline Female & 15 \\
\hline \multicolumn{2}{|l|}{ Age } \\
\hline$\geq 60$ & 21 \\
\hline $45-60$ & 11 \\
\hline$<45$ & 8 \\
\hline \multicolumn{2}{|l|}{ Tumor differentiation } \\
\hline Poor & 17 \\
\hline Moderate & 10 \\
\hline Well & 13 \\
\hline \multicolumn{2}{|l|}{ TNM stage } \\
\hline $\mathrm{T} 1+\mathrm{T} 2$ & 21 \\
\hline $\mathrm{T} 3+\mathrm{T} 4$ & 19 \\
\hline \multicolumn{2}{|c|}{ Lymph node metastases } \\
\hline Yes & 12 \\
\hline No & 28 \\
\hline \multicolumn{2}{|l|}{ Clinical stage } \\
\hline $\mathrm{I}+\mathrm{II}$ & 17 \\
\hline III+IV & 23 \\
\hline \multicolumn{2}{|l|}{ Site } \\
\hline Gingiva & 2 \\
\hline Tongue & 18 \\
\hline Buccal & 8 \\
\hline The floor of the oral & 2 \\
\hline Palate & 2 \\
\hline
\end{tabular}

ANT, adjacent non-cancerous tissues. OSCC, oral squamous cell carcinoma.

for qPCR. The PCR steps included $95^{\circ} \mathrm{C}$ predegeneration for $90 \mathrm{sec}$, followed by 40 amplification cycles of denaturation at $95^{\circ} \mathrm{C}$ for $10 \mathrm{sec}$ and annealing/elongation at $60^{\circ} \mathrm{C}$ for $30 \mathrm{sec}$. The transcript level of each gene was normalized to the expression of GAPDH. The comparative threshold cycle method $\left(2^{-\Delta \Delta C t}\right)$ was used to calculate relative changes in expression. For every sample, the experiment was repeated three times.

Western blot analysis. OSCC and cancer-adjacent oral mucosa tissues were weighed and ground into tissue powder in liquid nitrogen. Total protein from the OSCC and cancer-adjacent tissues was extracted using RIPA lysate (strong) (CWBio) containing a protease inhibitor cocktail (CWBio). Protein concentrations were measured using a BCA protein assay kit (CWBio). Subsequently, equal amounts of protein $(50 \mu \mathrm{g})$ were separated by SDS-PAGE and electrophoretically transferred onto PVDF membranes (Solarbio). The PVDF
Table IV. Primer sequences and product size used for reverse transcription-quantitative PCR.

\begin{tabular}{llc}
\hline Gene & \multicolumn{1}{c}{ Primer sequence } & $\begin{array}{c}\text { Product } \\
\text { size } \\
\text { (bp) }\end{array}$ \\
\hline PER2 & F: 5'-GCGTGTTCCACAGTTTCACC-3' & 146 \\
& R: 5'-GCGGATTTCATTCTCGTGGC-3' & \\
PIK3CA & F: 5'-GGTTTTGCTGTTCGGTGCTT-3' & 220 \\
& R: 5'-GGCCAAACCTCTGGCTAACT-3' & \\
PTEN & F: 5'-CTCAGCCGTTACCTGTGTGT-3' & 129 \\
& R: 5'-AGGTTTCCTCTGGTCCTGGT-3' & \\
P53 & F: 5'-ACCTATGGAAACTACTTCCTGAAA-3' & 257 \\
& R: 5'-GCTGCCCTGGTAGGTTTTCT-3' & \\
P14ARF & F: 5'-GTTTTCGTGGTTCACATCCCG-3' & 101 \\
& R: 5'-AGACGCTGGCTCCTCAGTA-3' & \\
Caspase-8 & F: 5'-CGCAAAGGAAGCAAGAACCC-3' & 209 \\
& R: 5'-GGCAGAAATTTGAGCCCTGC-3' & \\
GAPDH & F: 5'-GGATTTGGTCGTATTGGGCG-3' & 171 \\
& R: 5'-CTTCCCGTTCTCAGCCTTGA-3' & \\
\hline
\end{tabular}

F, forward. R, reverse.

membranes were then blocked with $5 \%$ skim milk for $1 \mathrm{~h}$ at room temperature, prior to being incubated with primary antibodies at $4^{\circ} \mathrm{C}$ overnight. Affinity-purified HRP-conjugated IgG secondary antibodies (1:4,500, CWBio) were incubated with the membranes in a $37^{\circ} \mathrm{C}$ incubator for $1 \mathrm{~h}$. An ECL-Advance Western Blot Detection system (Bio-Rad) was used for detection of the protein bands. Each experiment was repeated three times.

Immunohistochemistry. The 'two-step method' immunohistochemistry method was used to analyze protein expression in the paraffin-embedded sections (thickness, $4 \mu \mathrm{m}$ ). The paraffin-embedded sections were placed in incubators at $60^{\circ} \mathrm{C}$ for $1 \mathrm{~h}$, then dewaxed and rehydrated by conventional protocols. The sections were then placed in citric acid solution ( $\mathrm{pH} \mathrm{6.0)}$, heated in an oven until boiling, then heated for $10 \mathrm{~min}$ at $95^{\circ} \mathrm{C}$ for antigen retrieval. The sections were then naturally cooled to the ambient room temperature. To eliminate endogenous peroxidase activity, the sections were incubated in $3 \% \mathrm{H}_{2} \mathrm{O}_{2}$ deionized water for $10 \mathrm{~min}$. PBS was then used to wash the sections ( $3 \times 3 \mathrm{~min})$. Blocking was performed with $5 \%$ normal goat serum for $20 \mathrm{~min}$, and the sections were subsequently incubated with primary antibodies (Table I) overnight at $4^{\circ} \mathrm{C}$. Biotin-labeled secondary antibodies (SP test kit; SP-9000; Beijing Zhongshan Golden Bridge Biotechnology Co., Ltd., Beijing, China) were incubated with the sections at room temperature for $10 \mathrm{~min}$. Following 3x3-min washes in PBS, Streptavidin-HRP (SP test kit; SP-9000; Beijing Zhongshan Golden Bridge Biotechnology Co., Ltd.) was added to the sections and incubated at room temperature for $15 \mathrm{~min}$. Further 3x3-min washes in PBS were performed. For microscopic examination, DAB developer was added and incubated 
Table V. Levels of mRNA expression of each gene in OSCC tissue and cancer-adjacent oral mucosa tissue.

\begin{tabular}{lcclcc}
\hline Gene & OSCC & ANT & $\mathrm{F}$ & $\mathrm{t}$ & P-value \\
\hline PER2 & $0.45 \pm 0.27$ & 1.00 & 7.28 & -4.00 & 0.007 \\
PIK3CA & $1.20 \pm 0.30$ & 1.00 & 4.214 & -1.35 & 0.310 \\
PTEN & $0.67 \pm 0.11$ & 1.00 & 4.47 & 6.18 & 0.025 \\
P53 & $0.69 \pm 0.16$ & 1.00 & 5.46 & 3.92 & 0.030 \\
P14ARF & $0.54 \pm 0.34$ & 1.00 & 7.38 & 2.67 & 0.039 \\
Caspase-8 & $0.61 \pm 0.11$ & 1.00 & 16.00 & 6.31 & 0.390 \\
\hline
\end{tabular}

ANT, adjacent non-cancerous tissues. OSCC, oral squamous cell carcinoma. P-values reflect the differences of each gene in mRNA expression levels between OSCC tissue and cancer-adjacent oral mucosa tissue analyzed with Student's t-test, $\mathrm{P}<0.05$ were considered statistically significant.

at room temperature for $3 \mathrm{~min}$. The sections were then stained with hematoxylin, dehydrated, cleared, and observed under a light microscope after mounting with neutral resin. Slides with PBS added instead of primary antibody were used as the negative control. The positive slide provided by the company was used as the positive control. Five high-power fields were examined randomly in each section.

Results were evaluated using semiquantitative method. Intensity of positive staining (no staining, 0 ; light yellow, 1; brown, 2; dark brown, 3) and the percentage of positive cells (number of positive cells $\leq 25 \%, 0 ; 26-50 \%, 1 ; 51-75 \%, 2$; $\geq 76 \%, 3$ ) were scored. The sum of the intensity of positive staining scores and the percentage of positive cells scores was used finally to evaluate PER 2 expression as follows: 0-1-negative (-), 2-3-weakly positive (+), 4-5-positive (++), and $\geq 6$-strongly positive $(+++)$. Weakly positive $(+)$, positive $(++)$ and strongly positive $(+++)$ were judged as positive expression.

Statistical analysis. All data were analyzed using SPSS 19.0 software (SPSS Inc., Chicago, IL, USA). The experimental data from the RT-qPCR and western blot analyses were expressed as the mean \pm standard deviation (mean $\pm \mathrm{SD}$ ). A Student's t-test was used to make comparisons between two groups. Immunohistochemical data were expressed as the proportion of specimens with positive expression, and a $\chi^{2}$ test was used to analyze the differences in protein expression between OSCC and cancer-adjacent oral mucosa. A Spearman's rank correlation analysis was used to analyze the correlation between the level of PER 2 and of other proteins. Survival curves were drawn using the Kaplan-Meier method, and log-rank was used to compare survival between PER2-positive and PER2-negative cases $(\alpha=0.05$, two-sided). $P<0.05$ was considered to indicate a statistically significant difference.

\section{Results}

RT- $q$ PCR analysis. To determine the PER2, PIK3CA, PTEN, P53, P14ARF and caspase- 8 mRNA expression levels in human OSCC, RT-qPCR was performed on paired OSCC tissues and adjacent non-cancerous tissues from 8 patients. The results

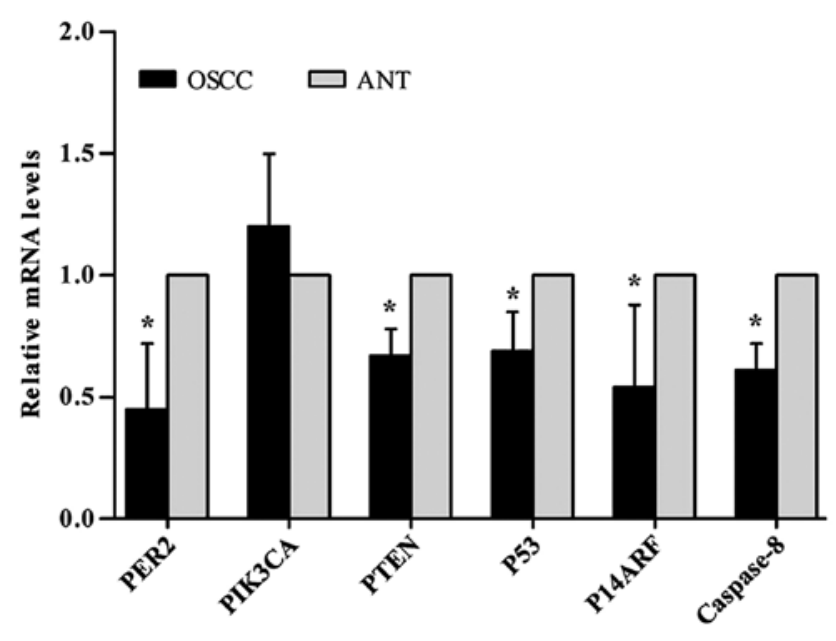

Figure 1. The mRNA expression levels of each gene in paired OSCC tissues and adjacent non-cancerous tissues. The mRNA expression levels of PER2, PIK3CA, PTEN, P53, P14ARF and caspase-8 in OSCC and ANT tissues (mean $\pm \mathrm{SD}$ ). The mRNA expression levels of PER2, PIK3CA, PTEN, P53, P14ARF and caspase-8 in OSCC group were downregulated in OSCC group compared to those in ANT group, while there was no notable difference of mRNA expression levels of PIK3CA between OSCC group and ANT group. Data are presented as the mean \pm SD. Differences between two groups were evaluated with Student's t-test. $\mathrm{P}<0.05$ was considered statistically significant.

Table VI. Results of protein average grey value in paired OSCC tissues and adjacent non-cancerous tissues.

\begin{tabular}{llcrc}
\hline Proteins & OSCC & ANT & t-test & P-value \\
\hline PER2 & $1.24 \pm 0.18$ & $6.17 \pm 0.55$ & 14.77 & 0.000 \\
PIK3CA & $0.12 \pm 0.021$ & $0.006 \pm 0.004$ & -9.42 & 0.001 \\
PTEN & $1.41 \pm 0.26$ & $3.33 \pm 0.37$ & 7.34 & 0.003 \\
P53 & $0.04 \pm 0.007$ & $1.20 \pm 0.08$ & 25.16 & 0.001 \\
P14ARF & $0.55 \pm 0.50$ & $1.19 \pm 0.08$ & 5.73 & 0.005 \\
Caspase-8 & $0.60 \pm 0.06$ & $2.93 \pm 0.29$ & 13.51 & 0.000 \\
\hline
\end{tabular}

Data are calculated by target protein average grey value/GAPDH protein average grey value. P-values reflect differences of each protein expression level among the two groups analyzed with Student's t-test, $\mathrm{P}<0.05$ was considered statistically significant.

revealed that the mRNA expression levels of PTEN, P53, P14ARF and caspase- 8 were reduced significantly in OSCC compared with adjacent non-cancerous tissues $(\mathrm{P}<0.05)$, whereas there were no significant differences in the PIK3CA mRNA expression levels, as shown in Table V and Fig. 1.

Western blot analysis. The PER2, PIK3CA, PTEN, P53, P14ARF and caspase- 8 protein expression levels were determined by western blotting in paired OSCC and adjacent non-cancerous tissues from 8 patients. The western blot results showed that the protein expression levels of PTEN, P53, P14ARF and caspase- 8 in OSCC were significantly reduced compared with those in non-cancerous tissues $(\mathrm{P}<0.05)$, while the PIK3CA protein level was significantly increased $(\mathrm{P}<0.05)$, as shown in Table VI, Figs. 2 and 3. 
Table VII. Result of immunohistochemical staining of each protein in OSCC tissues and cancer-adjacent tissues.

\begin{tabular}{lcllllll}
\hline & & \multicolumn{5}{c}{ Positive (percentage) } \\
\cline { 3 - 7 } Group & Cases & \multicolumn{1}{c}{ PER2 } & PIK3CA & PTEN & \multicolumn{1}{c}{ P53 } & P14ARF & Caspase-8 \\
\hline ANT & 14 & $13(92.9 \%)$ & $8(57.1 \%)$ & $12(85.7 \%)$ & $1(7.1 \%)$ & $14(100 \%)$ & $11(78.6 \%)$ \\
OSCC & 40 & $16(40 \%)$ & $37(92.5 \%)$ & $19(47.5 \%)$ & $17(42.5 \%)$ & $4(35 \%)$ & $10(25.0 \%)$ \\
OSCC:ANT & $\chi^{2}$ & $9.62^{\mathrm{a}}$ & $6.96^{\mathrm{a}}$ & $4.73^{\mathrm{a}}$ & $8.71^{\mathrm{a}}$ & $15.04^{\mathrm{a}}$ & $10.37^{\mathrm{a}}$ \\
\hline
\end{tabular}

${ }^{a} \mathrm{P}<0.05$. $\mathrm{P}$-values reflect differences in each protein expression positive rate by immunohistochemical staining among the two groups analyzed with $\chi^{2}$ test, $\mathrm{P}<0.05$ was considered statistically significant.

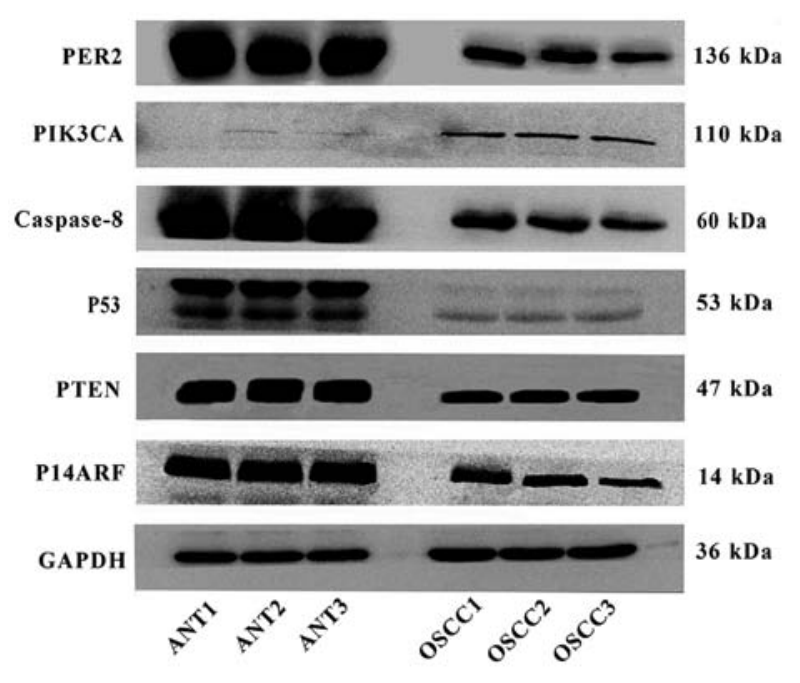

Figure 2. The results of each protein's ratio of the average grey value in paired OSCC tissues and adjacent non-cancerous tissues. The results are presented as the mean \pm SD. Differences between two groups of each protein expression were evaluated with Student's t-test. $\mathrm{P}<0.05$ was considered statistically significant.

Immunohistochemical analysis. The protein expression levels of PER2, PIK3CA, PTEN, P53, P14ARF and caspase-8 were analyzed in 40 OSCC tissues and 14 adjacent noncancerous tissues by immunohistochemical staining. The semi-quantified results of the immunohistochemical staining are presented in Table VII. The rates of positive expression of PER2, PTEN, P53, P14ARF and caspase- 8 proteins in OSCC were significantly lower than those in the adjacent non-cancerous tissues $(\mathrm{P}<0.05)$, while the rates of positive expression of PIK3CA and P53 proteins in OSCC were markedly higher than those in adjacent non-cancerous tissues $(\mathrm{P}<0.05)$. Representative immunohistochemical staining of PIK3CA, PTEN, P53, P14ARF and caspase- 8 proteins is shown in Fig. 4. Additionally, PER2 protein was mainly located in the cytoplasm in adjacent non-cancerous tissues, while it appeared to be expressed in both the cytoplasm and the cell nucleus in OSCC tissues according to subcellular localization analysis. The staining of PER 2 in the nuclei of the basal layer of epithelial tissue was more intense than that in the subcutaneous layer of cells, as shown in Fig. 4.

Low expression of PER2 is associated with the clinicopathological parameters of patients with OSCC. There was a clear

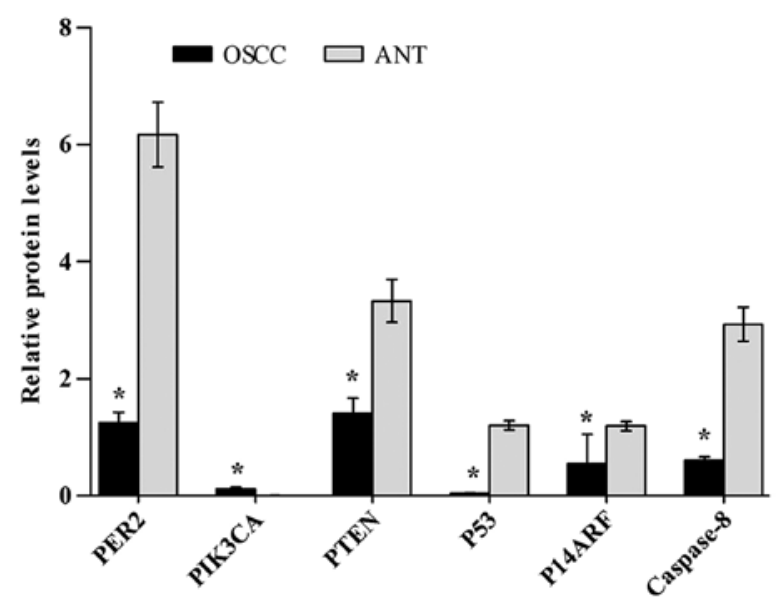

Figure 3. Western blot analysis of each protein expression levels in patients with OSCC. The level of GAPDH in each sample was detected as an internal control. One representative of three independent experiments with similar results is shown. ANT1, ANT2 and ANT3, each protein expression in adjacent non-cancerous tissues get from 3 patient with OSCC. OSCC1, OSCC2 and OSCC 3 , each protein expression in cancerous tissues get from 3 patient with OSCC.

association between PER2 expression and the clinical stage of OSCC: positive PER2 expression was markedly more frequent in OSCC of stages I/II than in OSCC of stages III/IV $(\mathrm{P}=0.000)$. Furthermore, PER2 expression was increased in OSCC of stages T1/T2 compared with that of stages T3/T4 $(\mathrm{P}=0.020)$, and was significantly more frequent in patients without lymphatic metastasis than in patients with lymphatic metastasis $(\mathrm{P}=0.049)$. There were no significant associations between PER2 and patient age, sex or pathological grade in OSCC $(\mathrm{P}<0.05)$, as shown in Table VIII.

Low expression of PER2 is associated with poor prognosis of patients with OSCC. Kaplan-Meier survival curves and log-rank tests demonstrated that OSCC patients with PER2-negative expression had a shorter overall survival time than those with PER2-positive expression ( $\mathrm{P}=0.044$ and $\mathrm{P}<0.05)$. The median post-operative survival times of patients with positive and negative PER 2 expression, respectively, were 62 and 32 months. Furthermore, the 3- and 5-year survival rates of patients with PER2-positive expression were $68.8 \%$ and $62.5 \%$, respectively, whereas the 3-year and 5-year survival rates of patients with PER2-negative expression were $41.7 \%$ and $29.2 \%$, respectively, as shown in Fig. 5. 

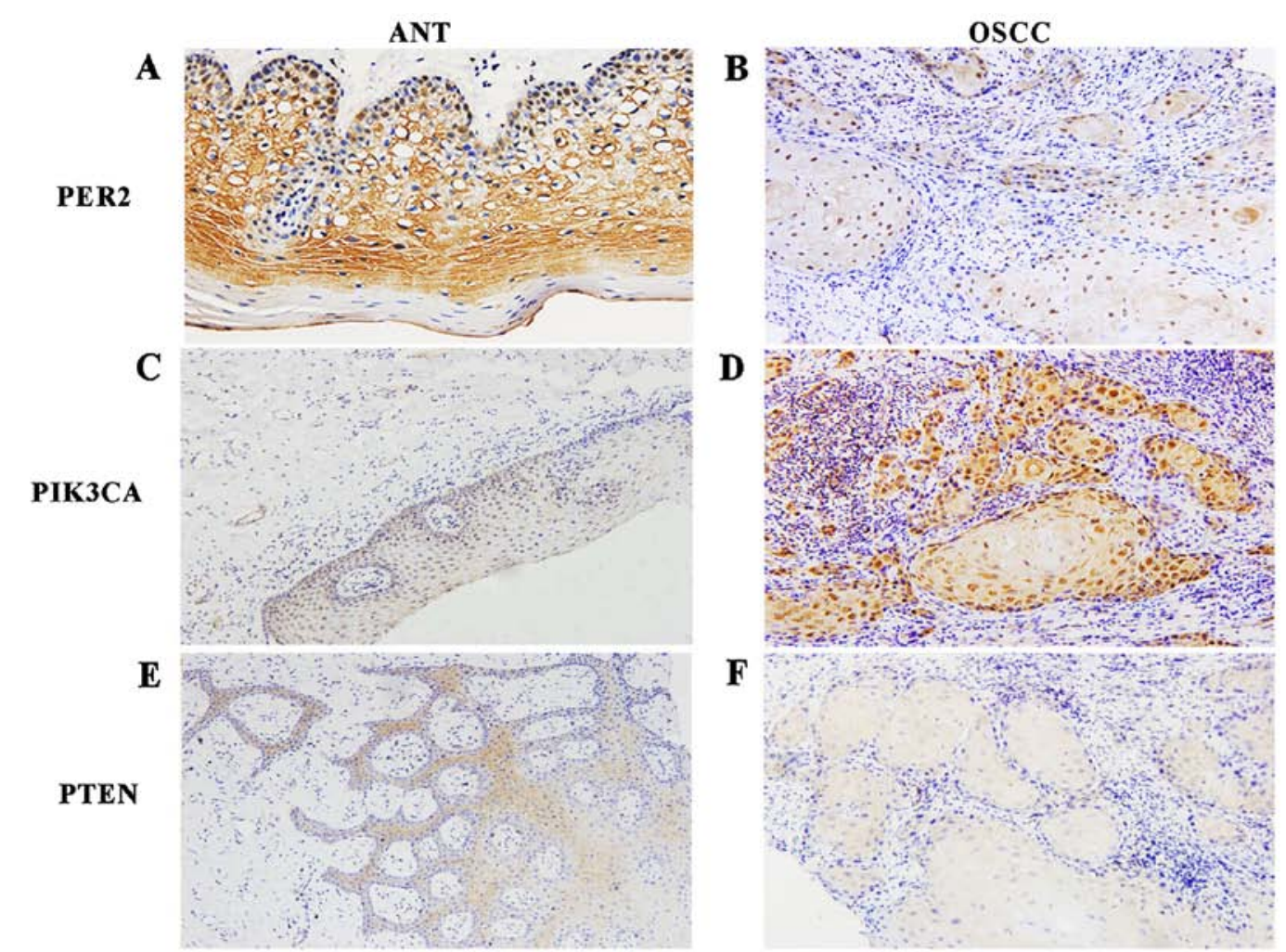

F
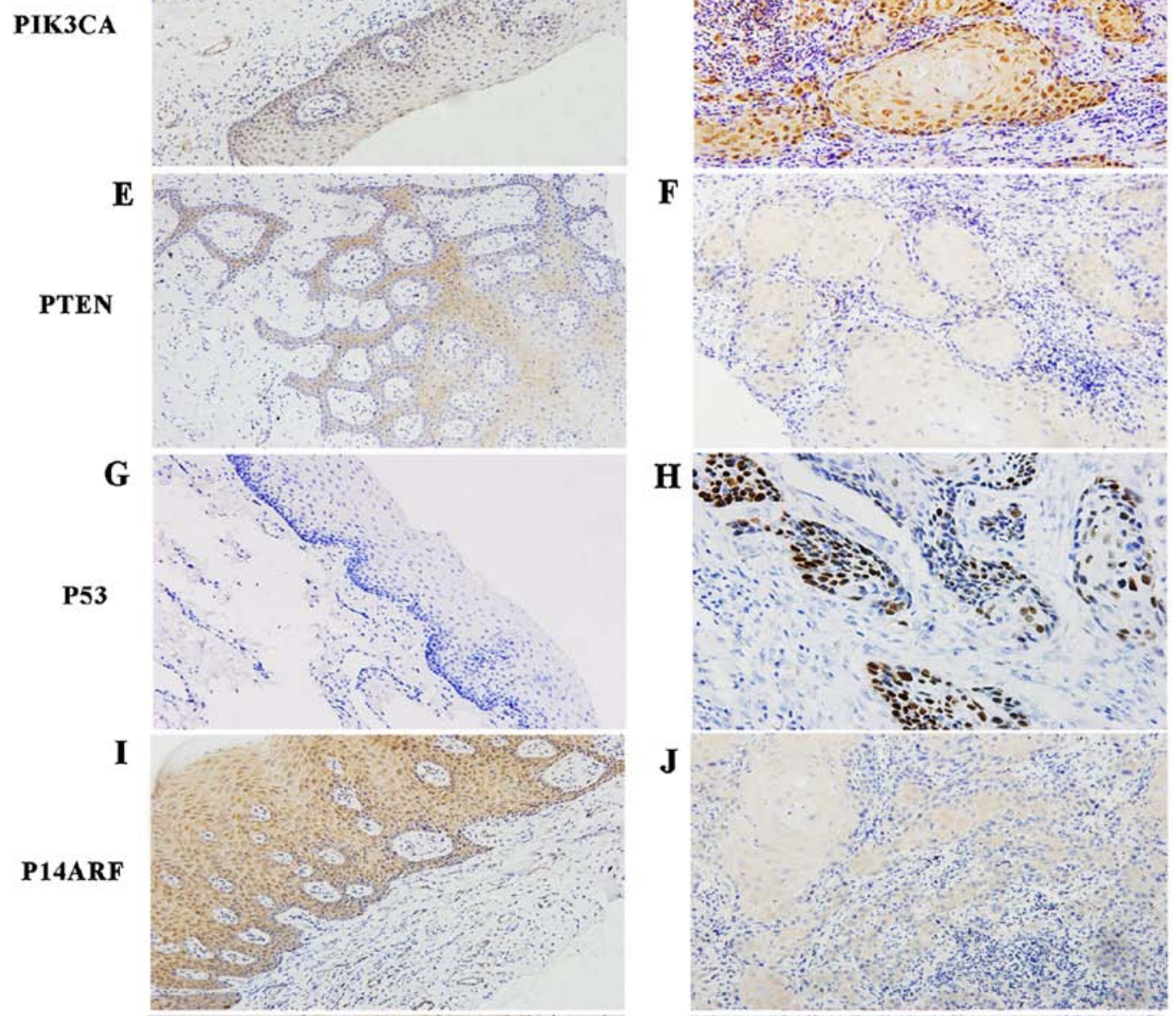

P14ARF
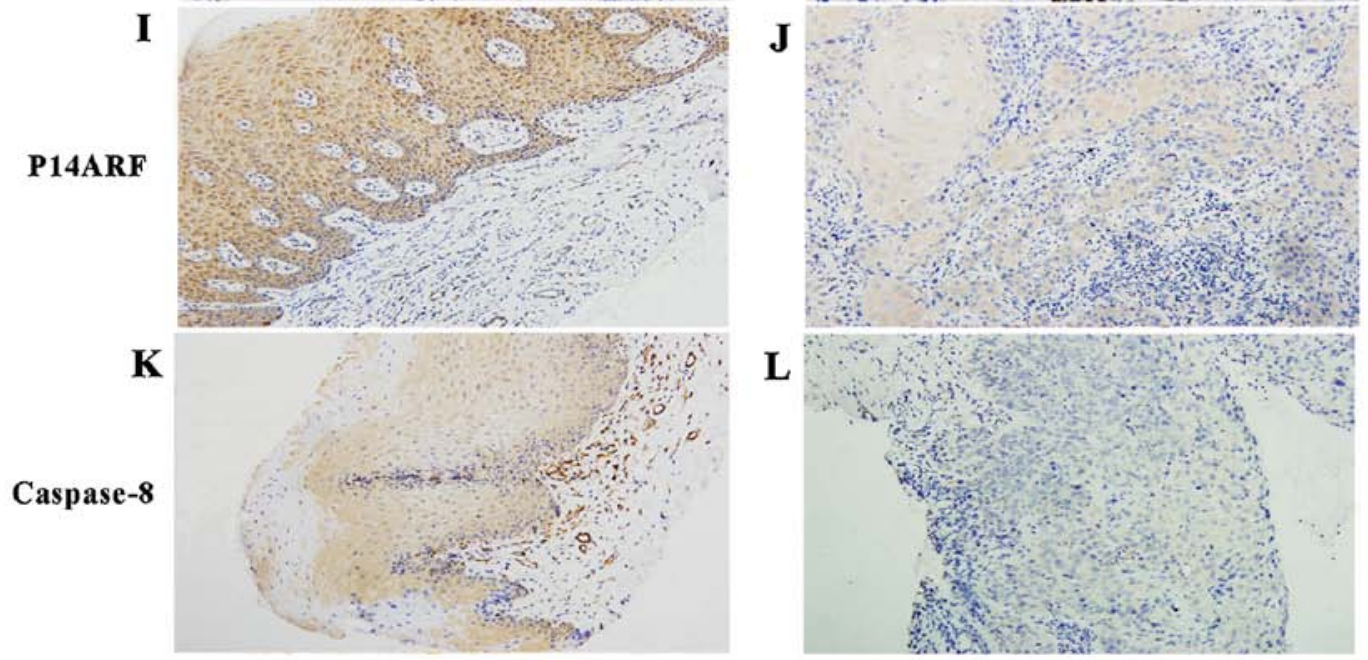

Figure 4. Immunohistochemical staining for each protein expression in tissues of OSCC. Patients (SP x200). (A) PER2 is positive (++) in ANT. (B) PER2 is weakly positive (+) in OSCC. (C) PIK3CA is weakly positive (+) in ANT. (D) PIK3CA is positive (++) in OSCC. (E) PTEN is positive (++) in ANT. (F) PTEN is positive (+) in OSCC. (G) P53 is negative (-) in ANT. (H) P53 is positive (++) in OSCC. (I) P14ARF is strong positive (+++) in ANT. (J) P14ARF is positive (++) in OSCC. (K) Caspase-8 is strong positive (++) in ANT. (L) Caspase-8 is weakly positive (+) in OSCC.

PER2 expression is associated with the expression of the tumor-related genes PIK3CA, PTEN, P53, P14ARF and caspase-8. PER 2 expression was negatively correlated with the expression of PIK3CA and P53 $(\mathrm{P}<0.05)$, and was positively correlated with the expression of PTEN, P14ARF and caspase-8 $(\mathrm{P}<0.05)$, as shown in Table IX.

\section{Discussion}

Previous studies have demonstrated that PER2 expression was decreased in gastric carcinoma, liver cancer, colon cancer and HNSCC (18-21). Similarly, this study also revealed that PER2 expression was reduced in OSCC compared with adjacent 


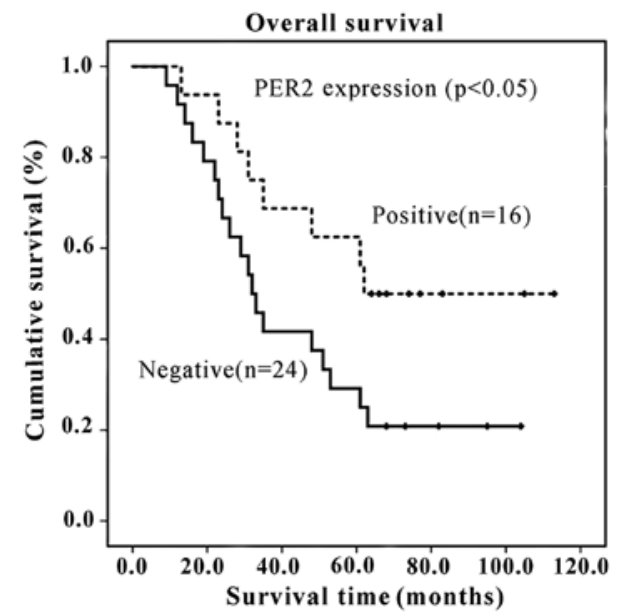

Figure 5. Kaplan-Meier survival curves for OSCC patients with PER2-positive expression (dotted line) versus PER2-negative expression (solid line). P-value reflects differences in overall survive rate between patients with positive and negative PER 2 expression evaluated with the $\chi^{2}$ test. $\mathrm{P}<0.05$ was considered statistically significant.

Table VIII. The expression of PER2 protein and its relationship with clinicopathological features of patients with OSCC.

\begin{tabular}{|c|c|c|c|c|}
\hline \multirow[b]{2}{*}{ Parameters } & \multirow[b]{2}{*}{ Case } & PER2 expression & & \\
\hline & & Negative Positive & $\chi^{2}$ & P-value \\
\hline
\end{tabular}

\begin{tabular}{lrrrrr}
\hline Tissue type & & & & & \\
OSCC & 40 & 24 & 16 & 11.653 & 0.001 \\
ANT & 14 & 1 & 13 & & \\
Age & & & & & \\
$\geq 60$ & 21 & 14 & 7 & 0.047 & 0.972 \\
$45-60$ & 11 & 7 & 4 & & \\
$<45$ & 8 & 5 & 3 & & \\
Sex & & & & & \\
Male & 25 & 16 & 9 & 0.029 & 0.864 \\
Female & 15 & 10 & 5 & &
\end{tabular}

Tumor

differentiation

$\begin{array}{lrrrrr}\text { Well } & 13 & 7 & 8 & 3.022 & 0.221 \\ \text { Moderate } & 10 & 6 & 4 & & \\ \text { Poor } & 17 & 13 & 4 & & \\ \text { T staging } & & & & & \\ \text { T1+T2 } & 21 & 9 & 12 & 5.414 & 0.020^{\mathrm{a}} \\ \text { T3+T4 } & 19 & 15 & 4 & 3.889 & 0.049^{\mathrm{a}}\end{array}$

Lymph node metastasis

$\begin{array}{lrrrrr}\text { No } & 28 & 14 & 14 & 3.889 & 0.049^{\mathrm{a}} \\ \text { Yes } & 12 & 10 & 2 & & \\ \text { Clinical stage } & & & & & \\ \text { I+II } & 17 & 2 & 15 & 28.62 & 0.000^{\mathrm{a}} \\ \text { III+IV } & 23 & 22 & 2 & & \end{array}$

ANT, adjacent non-cancerous tissues. OSCC, oral squamous cell car-

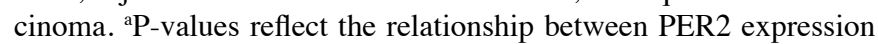
and clinicopathological features with $\chi^{2}$ test. $\mathrm{P}<0.05$ were considered statistically significant.
Table IX. Spearman's rank correlation analysis between PER2 protein and tumor related protein in patients with OSCC.

\begin{tabular}{|c|c|c|c|c|c|c|c|}
\hline \multirow[b]{2}{*}{ Protein } & \multirow[b]{2}{*}{$\mathrm{N}$} & \multicolumn{4}{|c|}{ PER2 } & \multirow[b]{2}{*}{$\mathrm{r}$} & \multirow[b]{2}{*}{ P-value } \\
\hline & & - & + & ++ & +++ & & \\
\hline PIK3CA & & & & & & -0.603 & $<0.01$ \\
\hline- & 3 & 0 & 1 & 0 & 2 & & \\
\hline+ & 10 & 5 & 5 & 4 & 0 & & \\
\hline++ & 10 & 9 & 1 & 0 & 0 & & \\
\hline+++ & 17 & 10 & 2 & 5 & 0 & & \\
\hline PTEN & & & & & & 0.442 & 0.004 \\
\hline- & 21 & 15 & 6 & 0 & 0 & & \\
\hline+ & 8 & 6 & 1 & 0 & 1 & & \\
\hline++ & 7 & 2 & 2 & 2 & 1 & & \\
\hline+++ & 4 & 1 & 0 & 2 & 1 & & \\
\hline P53 & & & & & & -0.397 & 0.011 \\
\hline- & 23 & 10 & 11 & 2 & 0 & & \\
\hline+ & 7 & 5 & 1 & 1 & 0 & & \\
\hline++ & 7 & 6 & 1 & 0 & 0 & & \\
\hline+++ & 3 & 3 & 0 & 0 & 0 & & \\
\hline P14ARF & & & & & & 0.317 & 0.047 \\
\hline- & 26 & 17 & 8 & 0 & 1 & & \\
\hline+ & 7 & 5 & 1 & 1 & 0 & & \\
\hline++ & 4 & 1 & 0 & 1 & 2 & & \\
\hline+++ & 3 & 1 & 0 & 2 & 0 & & \\
\hline Caspase- 8 & & & & & & 0.620 & $<0.01$ \\
\hline- & 30 & 23 & 5 & 1 & 1 & & \\
\hline+ & 6 & 1 & 2 & 2 & 1 & & \\
\hline++ & 2 & 0 & 1 & 1 & 0 & & \\
\hline+++ & 2 & 0 & 1 & 0 & 1 & & \\
\hline
\end{tabular}

$r$ shows the correlation coefficient, the greater the absolute value of correlation coefficient indicates the related degree is higher; + , positive correlation; -, negative correlation; $\mathrm{P}<0.05$ were considered statistically significant.

non-cancerous tissue. Further analysis found that the expression of PER2 was associated with the clinical stage of OSCC, as well as the lymphatic metastasis status and the patient survival time: reduced expression of PER 2 could promote the occurrence and metastasis of OSCC and shorten survival time. This seems to be consistent with research results of Zhao et al in gastric carcinoma (18) and of Wang et al in colon cancer (20).

The specific molecular mechanisms by which an abnormal expression level of PER2 promotes the occurrence and development of OSCC has remained unclear until now. P53 and P14ARF are known to play important roles in the occurrence and development of OSCC $(32,33)$. P14ARF can restrain MDM2, thereby relieving the ubiquitination and degradation of P53 and increasing the intracellular level of functional P53 (35). P14ARF can thus positively regulate P53, and can also form a feedback loop (35). Loss of p53 in OSCC may be partly attributed to reduced P14ARF expression. Previous evidence has shown that wild-type P53 (wt-P53) functions as 
a tumor suppressor, whereas mutant type P53 (mt-P53) does not (36). Gröbe et al and Friedrich et al reported that mt-P53 accounted for 40-60\% of all P53 in OSCC $(37,38)$. wt-p53 protein's half-life is very short, its detection is difficult by general experimental methods. However mt-p53 protein is more stable. mt-p53 protein can be detected by immunohistochemistry. Although weaker staining of p53 protein might be detected due to increased stability when cells are in a state of oxidative cellular stress, it is generally believed that oxidative cellular stress had very little influence on the positive rate of p53 when detected by immunohistochemistry. Judging mutation status of p53 according to the result of positive expression detected by immunohistochemistry with highly specificity and sensitivity (39). Our results of immunohistochemistry staining show that the p53-positive expression rate in OSCC was $42.5 \%$, which could be considered roughly representing its mutation status in OSCC. Further analysis show that PER2 was negatively correlated with p53 positive expression, and positively correlated with P14ARF in OSCC.

Studies have revealed that PIK3CA and PTEN have an important role in the occurrence and development of OSCC (23-31). PIK3CA is the key molecule in the PI3K/AKT signaling pathway (40), while PTEN is the most important negative regulatory component in the PI3K/AKT signaling pathway (41). Abnormal activation of PI3K/AKT signaling may be associated with the functional loss of PTEN (42). According to the study by Chen et al, the downregulation of PER2 in cisplatin-resistant lung cancer A549/DDP cells resulted in increased PI3K/AKT/mTOR pathway activity. Conversely, after upregulating PER2, PI3K/AKT/mTOR pathway activity was reduced, thereby promoting apoptosis (43). Wang et al injected a recombinant PER2 plasmid to induce PER2 overexpression in an allograft ovarian cancer nude mouse model, revealing that PIK3CA and AKT protein expression levels could be reduced as a result (44). The present study also indicated that PER2 expression in clinical specimens of OSCC was negatively correlated with PIK3CA expression, but positively correlated with PTEN expression. Therefore, there may be an interaction between PER 2 and the PI3K/AKT signaling pathway. PER 2 could restrain the activity of the PI3K signaling pathway through two modes: firstly, PER2 might restrain PTEN expression, so as to weaken the negative regulatory effect of PTEN on PI3K/AKT signaling, thus indirectly activating PI3K/AKT signaling; secondly, PER2 might interact with the P110 subunits, encoded by PIK3CA, in the PI3K/AKT signaling pathway, so as to restrain the intracellular conduction of extracellular signals. The molecular mechanism by which PER2 regulates PI3K/AKT should be further studied.

Our previous studies found that downregulating PER2 in OSCC cells could markedly reduce cell apoptosis $(25,26)$. Caspase- 8 is an important apoptosis-related gene, and its abnormal expression is closely associated with carcinogenesis (45). Previous studies have already demonstrated decreased caspase- 8 expression in various types of cancers $(34,46)$. Consistently, the results of the current study also found that caspase- 8 mRNA and protein expression levels in OSCC were reduced, and further demonstrated a positive correlation between PER 2 and caspase- 8 . These findings suggest that the abnormally decreased expression of PER2 may reduce apoptosis by regulating caspase- 8 , thereby leading to carcinogenesis.

In the present study, a subcellular localization analysis was conducted. We found that PER2 staining was mainly present in the cytoplasm in cancer-adjacent oral mucosa cells, while OSCC cells exhibited both cytoplasmic and nuclear PER2 staining. We also found that PER2 staining in the nuclei of stratum basale cells was more intense than that in the nuclei of stratum spinosum and granular layer cells. Ectopic expression of PER2 in cancer cells may promote cell proliferation, and this may be one of the causes of tumor occurrence and development.

In conclusion, the expression of PER2 is reduced in OSCC. Reduced expression of PER2 is closely associated with the occurrence and development of OSCC, and may therefore be an important biological marker to predict OSCC prognosis. PER2 may exert its antitumor effect via the P53/P14ARF, PIK3CA/AKT and caspase- 8 pathways. These findings suggest that PER2 may be an important target for cancer therapy; however, the exact role of PER2 must be further verified. Future studies should explore the key molecules that interact with PER2 in the aforementioned pathways, and investigate their molecular mechanisms.

\section{Acknowledgements}

This study was supported by the Program for Graduate Student Research Innovation in Chongqing (CYS16168).

\section{References}

1. Dibner C, Schibler U and Albrecht U: The mammalian circadian timing system: Organization and coordination of central and peripheral clocks. Annu Rev Physiol 72: 517-549, 2010.

2. Welsh DK, Yoo SH, Liu AC, Takahashi JS and Kay SA: Bioluminescence imaging of individual fibroblasts reveals persistent, independently phased circadian rhythms of clock gene expression. Curr Biol 14: 2289-2295, 2004.

3. Takahashi JS: Transcriptional architecture of the mammalian circadian clock. Nat Rev Genet 18: 164-179, 2017.

4. Miller BH, McDearmon EL, Panda S, Hayes KR, Zhang J, Andrews JL, Antoch MP, Walker JR, Esser KA, Hogenesch JB, et al: Circadian and CLOCK-controlled regulation of the mouse transcriptome and cell proliferation. Proc Natl Acad Sci USA 104: 3342-3347, 2007.

5. Matsuo T, Yamaguchi S, Mitsui S, Emi A, Shimoda F and Okamura $\mathrm{H}$ : Control mechanism of the circadian clock for timing of cell division in vivo. Science 302: 255-259, 2003.

6. Zhu Z, Hua B, Shang Z, Yuan G, Xu L, Li E, Li X, Sun N, Yan Z, Qian R, et al: Altered clock and lipid metabolism-related genes in atherosclerotic mice kept with abnormal lighting condition. BioMed Res Int 2016: 5438589, 2016.

7. Di Cara F and King-Jones $\mathrm{K}$ : The circadian clock is a key driver of steroid hormone production in Drosophila. Curr Biol 26: 2469-2477, 2016.

8. Li J, Terry EE, Fejer E, Gamba D, Hartmann N, Logsdon J, Michalski D, Rois LE, Scuderi MJ, Kunst M, et al: Achilles is a circadian clock-controlled gene that regulates immune function in Drosophila. Brain Behav Immun 61: 127-136, 2017.

9. Tao H, Li X, Qiu JF, Cui WZ, Sima YH and Xu SQ: Inhibition of expression of the circadian clock gene period causes metabolic abnormalities including repression of glycometabolism in Bombyx mori cells. Sci Rep 7: 46258, 2017.

10. Vieira E, Ruano E, Figueroa AL, Aranda G, Momblan D, Carmona F, Gomis R, Vidal J and Hanzu FA: Altered clock gene expression in obese visceral adipose tissue is associated with metabolic syndrome. PLoS One 9: e111678, 2014. 
11. Akashi M, Matsumura R, Matsuo T, Kubo Y, Komoda H and Node K: Hypercholesterolemia causes circadian dysfunction: A potential risk factor for cardiovascular disease. EBioMedicine 20 127-136, 2017.

12. Wood PA, Yang $X$ and Hrushesky WJ: Clock genes and cancer. Integr Cancer Ther 8: 303-308, 2009.

13. Zheng B, Larkin DW, Albrecht U, Sun ZS, Sage M, Eichele G, Lee CC and Bradley A: The mPer2 gene encodes a functional component of the mammalian circadian clock. Nature 400: 169-173, 1999.

14. Albrecht U, Bordon A, Schmutz I and Ripperger J: The multiple facets of Per2. Cold Spring Harb Symp Quant Biol 72: 95-104 2007.

15. Toh KL, Jones CR, He Y, Eide EJ, Hinz WA, Virshup DM, Ptácek LJ and $\mathrm{Fu} \mathrm{YH}$ : An hPer2 phosphorylation site mutation in familial advanced sleep phase syndrome. Science 291: 1040-1043, 2001

16. Fu L, Pelicano H, Liu J, Huang P and Lee C: The circadian gene Period2 plays an important role in tumor suppression and DNA damage response in vivo. Cell 111: 41-50, 2002.

17. Wood PA, Yang X, Taber A, Oh EY, Ansell C, Ayers SE, Al-Assaad Z, Carnevale K, Berger FG, Peña MM, et al: Period 2 mutation accelerates ApcMin/+ tumorigenesis. Mol Cancer Res 6: 1786-1793, 2008

18. Zhao H, Zeng ZL, Yang J, Jin Y, Qiu MZ, Hu XY, Han J, Liu KY, Liao JW, Xu RH, et al: Prognostic relevance of Period1 (Per1) and Period2 (Per2) expression in human gastric cancer. Int J Clin Exp Pathol 7: 619-630, 2014

19. Lin YM, Chang JH, Yeh KT, Yang MY, Liu TC, Lin SF, Su WW and Chang JG: Disturbance of circadian gene expression in hepatocellular carcinoma. Mol Carcinog 47: 925-933, 2008.

20. Wang Y, Hua L, Lu C and Chen Z: Expression of circadian clock gene human Period2 (hPer2) in human colorectal carcinoma. World J Surg Oncol 9: 166, 2011

21. Hsu CM, Lin SF, Lu CT, Lin PM and Yang MY: Altered expression of circadian clock genes in head and neck squamous cell carcinoma. Tumour Biol 33: 149-155, 2012

22. Hua H, Wang Y, Wan C, Liu Y, Zhu B, Yang C, Wang X, Wang Z Cornelissen-Guillaume $\mathrm{G}$ and Halberg F: Circadian gene mPer2 overexpression induces cancer cell apoptosis. Cancer Sci 97: 589-596, 2006

23. Cheng AY, Zhang Y, Mei HJ, Fang S, Ji P, Yang J, Yu L and Guo WC: Construction of a plasmid for overexpression of human circadian gene period 2 and its biological activity in osteosarcoma cells. Tumour Biol 36: 3735-3743, 2015.

24. Oda A, Katayose Y, Yabuuchi S, Yamamoto K, Mizuma M, Shirasou S, Onogawa T, Ohtsuka H, Yoshida H, Hayashi H, et al: Clock gene mouse period2 overexpression inhibits growth of human pancreatic cancer cells and has synergistic effect with cisplatin. Anticancer Res 29: 1201-1209, 2009

25. Wang Q, Ao Y, Yang K, Tang H and Chen D: Circadian clock gene Per2 plays an important role in cell proliferation, apoptosis and cell cycle progression in human oral squamous cell carcinoma. Oncol Rep 35: 3387-3394, 2016.

26. Su X, Chen D, Yang K, Zhao Q, Zhao D, Lv X and Ao Y: The circadian clock gene PER2 plays an important role in tumor suppression through regulating tumor-associated genes in human oral squamous cell carcinoma. Oncol Rep 38: 472-480, 2017.

27. Chi AC, Day TA and Neville BW: Oral cavity and oropharyngeal squamous cell carcinoma - an update. CA Cancer J Clin 65 401-421, 2015

28. Wan X, Li X, Yang J, Lv W, Wang Q, Chen Y and Li Y: Genetic association between PIK3CA gene and oral squamous cell carcinoma: A case control study conducted in Chongqing, China. Int J Clin Exp Pathol 8: 13360-13366, 2015.
29. Chen Y, Hou Q, Yan W, Luo J, Chen D, Liu Z, He S and Ding X: PIK3CA is critical for the proliferation, invasiveness, and drug resistance of human tongue carcinoma cells. Oncol Res 19: 563-571, 2011.

30. Jasphin SS, Desai D, Pandit S, Gonsalves NM, Nayak PB and Iype A: Immunohistochemical expression of phosphatase and tensin homolog in histologic gradings of oral squamous cell carcinoma. Contemp Clin Dent 7: 524-528, 2016.

31. Rahmani A, Alzohairy M, Babiker AY, Rizvi MA and Elkarimahmad HG: Clinicopathological significance of PTEN and bcl 2 expressions in oral squamous cell carcinoma. Int $\mathrm{J}$ Clin Exp Pathol 5: 965-971, 2012.

32. Mao C, Lu Y, Lai Q, Xia Y and Yang C: Expression of p53 gene in oral squamous cell carcinoma and its relation with clinical and pathological parameters and prognosis of patients. Chin Med Sci J 10: 199-203, 1995

33. Shintani S, Nakahara $Y$, Mihara M, Ueyama $Y$ and Matsumura T: Inactivation of the p14(ARF), p15(INK4B) and p16(INK4A) genes is a frequent event in human oral squamous cell carcinomas. Oral Oncol 37: 498-504, 2001.

34. Elrod HA, Fan S, Muller S, Chen GZ, Pan L, Tighiouart M, Shin DM, Khuri FR and Sun SY: Analysis of death receptor 5 and caspase- 8 expression in primary and metastatic head and neck squamous cell carcinoma and their prognostic impact. PLoS One 5: e12178, 2010.

35. Lin J and Zhu MH: Interactive pathway of ARF-mdm2-p53. Ai Zheng 22: 328-330, 2003 (In Chinese).

36. Liu J, Zhang C and Feng Z: Tumor suppressor p53 and its gain-of-function mutants in cancer. Acta Biochim Biophys Sin (Shanghai) 46: 170-179, 2014

37. Gröbe A, Hanken H, Al-Dam A, Cachovan G, Smeets R, Krohn A, Clauditz T, Grob T, Simon R, Sauter G, et al: P53 immunohistochemical expression does not correlate with clinica features in 207 carcinomas of the oral cavity and in the head and neck region. Clin Oral Investig 18: 211-217, 2014.

38. Friedrich RE, Giese M, Riethdorf S and Loning T: P53-mutation in smears of oral squamous cell carcinoma. Anticancer Res 20D: 4927-4930, 2000.

39. Takami H, Yoshida A, Fukushima S, Arita H, Matsushita Y, Nakamura T, Ohno M, Miyakita Y, Shibui S, Narita Y, et al: Revisiting TP53 mutations and immunohistochemistry - A comparative study in 157 diffuse gliomas. Brain Pathol 25: 256-265, 2015

40. Lai K, Killingsworth MC and Lee CS: Gene of month: PIK3CA. J Clin Pathol 68: 253-257, 2015.

41. Lee JO, Yang H, Georgescu MM, Di Cristofano A, Maehama T, Shi Y, Dixon JE, Pandolfi P and Pavletich NP: Crystal structure of the PTEN tumor suppressor: Implications for its phosphoinositide phosphatase activity and membrane association. Cell 99: 323-334, 1999.

42. Di Cristofano A and Pandolfi PP: The multiple roles of PTEN in tumor suppression. Cell 100: 387-390, 2000.

43. Chen B, Tan Y, Liang Y, Li Y, Chen L, Wu S, Xu W, Wang Y, Zhao $\mathrm{W}$ and Wu J: Per2 participates in AKT-mediated drug resistance in A549/DDP lung adenocarcinoma cells. Oncol Lett 13: 423-428, 2017.

44. Wang Z, Li L and Wang Y: Effects of Per2 overexpression on growth inhibition and metastasis, and on MTA1, nm23-H1 and the autophagy-associated PI3K/PKB signaling pathway in nude mice xenograft models of ovarian cancer. Mol Med Rep 13 4561-4568, 2016

45. Fulda S: Caspase- 8 in cancer biology and therapy. Cancer Lett 281: 128-133, 2009

46. Wang X, Fu Z, Chen Y and Liu L: Fas expression is downregulated in gastric cancer. Mol Med Rep 15: 627-634, 2017. 